\title{
DEGREE OF ASSOCIATION BETWEEN FLUDEOXYGLUCOSE UPTAKE IN PALATINE TONSILS IN PET SCAN AND HISTOPATHOLOGIC EXAMINATION IN FOLLOW UP CASES OF NON-HODGKIN'S LYMPHOMA AND HODGKIN'S LYMPHOMA
}

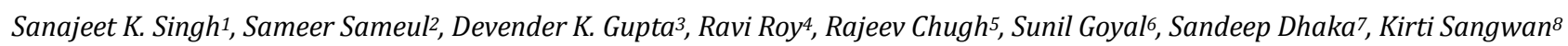

1 Professor and HOD, Department of ENT, HNS Army Hospital $R \&$ R, New Delhi, India.

${ }^{2} 2^{\text {nd }}$ Year Resident, Department of ENT, HNS Army Hospital R \& R, New Delhi, India.

${ }^{3}$ Assistant Professor, Department of ENT, HNS Army Hospital $R \&$ R, New Delhi, India.

${ }^{4}$ Associate Professor, Department of ENT, HNS Army Hospital R \& R, New Delhi, India.

${ }^{5}$ Associate Professor, Department of ENT, HNS Army Hospital R \& R, New Delhi, India.

${ }^{6}$ Assistant Professor, Department of ENT, HNS Army Hospital $R \& R$, New Delhi, India.

${ }^{7} 1^{\text {st }}$ Year Resident, Department of ENT, HNS Army Hospital R \& R, New Delhi, India.

${ }_{81}^{8}$ st Year Resident, Department of ENT, HNS Army Hospital R \& R, New Delhi, India.

\begin{abstract}
BACKGROUND

Lymphomas are heterogeneous group of malignancies that has distinct biological behaviour. FDG-PET is the investigation of choice used to stage and restage lymphoma patients. False-positive results are possible, because of infection, inflammation, hypermetabolic brown fat surrounding lymph nodes or reactive process producing strong 18F-FDG uptake. In these cases, and not uncommonly after chemotherapy, reactive enlargement of the lymph nodes, tonsils, and thymus gland can be detected.
\end{abstract}

\section{METHODS}

Prospective study of 30 follow up patients who underwent chemotherapy for lymphoma showing FDG uptake with standardized uptake value max of 5.0 and above on PET scan in palatine tonsil.

\section{RESULTS}

In our study, we found that association between FDG uptake on PET scan and relapse on histopathological examination was not significant at $\mathrm{p}$ value of 0.084 .

\section{CONCLUSIONS}

We found no significant relationship between increase in FDG uptake on PET scan and relapse, in palatine tonsils, in follow up cases of lymphoma, following histopathology results.

HOW TO CITE THIS ARTICLE: Singh SK, Samuel S, Gupta DK, et al. Degree of association between fludeoxyglucose uptake in palatine tonsils in pet scan and histopathologic examination in follow up cases of non-Hodgkin's lymphoma and Hodgkin's lymphoma. J. Evolution Med. Dent. Sci. 2019;8(16):1298-1300, DOI: 10.14260/jemds/2019/289

\section{BACKGROUND}

Lymphomas are heterogeneous group of malignancies that has distinct biological behaviour according to the subtype and the degree of differentiation.

Lymphomas can be divided into two main groups: Hodgkin's (HL) and non-Hodgkin (NHL). The most common type is NHL which represents approximately $85 \%$ of all lymphomas. FDG-PET is the investigation of choice used to stage and restage HL and NHL. Staging HL and NHL with FDGPET is directly dependent on many different factors including biological and technical aspects of the lymphoma.

One of the most important factors is the histology. Normally, the most common subtypes of HL and the most aggressive NHL, such as diffuse large B cell lymphoma (DLBCL), show high levels of cell proliferation that reflect in a higher aerobic glycolysis rate

'Financial or Other Competing Interest': None.

Submission 10-11-2018, Peer Review 10-04-2019,

Acceptance 17-04-2019, Published 22-04-2019.

Corresponding Author:

Dr. Sameer Samuel,

Resident,

Department of ENT,

HNS Army Hospital R \& R,

New Delhi, India.

E-mail: samuelsameer@yahoo.com

DOI: $10.14260 /$ jemds $/ 2019 / 289$
Therefore, higher FDG uptake values are seen in aggressive tumours compared to more indolent ones that usually show faint or very low glucose concentrations.

The level of FDG uptake can be evaluated semiquantitatively using the standardized uptake value (SUV). The SUV is the activity in the lesion measured as $\mu \mathrm{Ci} / \mathrm{mL}$ corrected for the patient's weight and the dose of FDG administered.

The 2-[fluorine-18] fluoro-2-deoxy-d-glucose positron emission tomography/computed tomography (FDG-PET/CT) has been effective for the diagnosis, staging, and restaging of malignancies of the head and neck region, including carcinoma, melanoma, and lymphoma. During the follow-up, negative FDG-PET/CT scans almost exclude a relapse of the disease, but 18F-FDG uptake does not necessarily means its returns.[1,2]

The degree of FDG uptake is often measured to allow comparison within and between different patients and diseases by the standardized uptake value (SUV).[3] In normal head and neck structures, 18F-FDG uptake is usually seen in the palatine tonsils, soft palate, and lingual tonsils. 18F-FDG accumulation in the major salivary glands, spinal cord, and vocal cords is variable; whereas in the inferior concha, thyroid gland, and tongue is typically minimal.[4] In lymphoma patients, unfortunately, false-positive results are possible, because an infection, inflammation, hypermetabolic 
brown fat surrounding lymph nodes or reactive process producing strong 18F-FDG uptake might be present. ${ }^{[2,5-8]}$ In these cases, and not uncommonly after chemotherapy, reactive enlargement of the lymph nodes, tonsils, and thymus gland can be detected. [8-10]

\section{METHODS}

We present a prospective study conducted between July 2015 and May 2017 in our institute Army Hospital Research and Referral of 30 Follow up Patients who underwent chemotherapy for lymphoma showing FDG Uptake with SUV max of 5.0 and above on PET scan in Palatine tonsils.

Sample was taken after doing a pilot study and convenient sampling was used. A well-designed structured questionnaire after pre testing \& validation was used to collect information.

PET scan was performed at our institute as per the protocol mentioned below-

Protocol for PET-Whole body PET/CT scan (base of skull to mid-thigh) was done 45 minutes after i.v. injection of 296 MBq of 18F-FDG, using a whole-body full ring dedicated LSO PET/CT scanner. CT images were obtained using $130 \mathrm{KV}$ and $90 \mathrm{mAs}$ (mean) without administration of intravenous or oral contrast. CT base attenuation correction was done. Images were reconstructed using standard iterative algorithm (OSEM) and reformatted into Transaxial, Coronal and Sagittal views. A 3D image and fusion images of PET \& CT were obtained.
All 30 patients underwent B/L Tonsillectomy and results of histopathology were corelated with PET scan findings.

\section{Statically Analysis}

The collected data was compiled \& tabulated using Microsoft Excel 2007 and analysed using SPSS Version 20. Descriptive statistical analysis was carried out in the present study. Significance was assessed at $5 \%$ level by using chi-square of significance.

\section{RESULTS}

In our study (July 2015 to May 2017) of 30 patients $66.7 \%$ were male and mean age was 31 (9-62 years), 14 with NHL and 16 with HL who underwent chemotherapy (46.6\% received ABVD**, $40 \%$ received $\mathrm{RCHOP*}, 13.3 \%$ received $\mathrm{R}$ $\mathrm{BM}^{* * *}$ ) for lymphoma. In follow up of all patients, FDG uptake was present with SUV of more than 5.0. PET Scan was conducted in our nuclear medicine department. All patients underwent B/L Tonsillectomy, On HPE, one patient was suspicious of follicular NHL, two patients with nodular sclerosing type and three patients were NHL -B cell type, in rest 24 patients, reactive hyperplasia was seen in lymph nodes.

${ }^{*} \mathrm{R}$ - Rituximab, C- Cyclophosphamide, H- Doxorubicin, OVincristine, P- Prednisolone

** A- Adriamycin, B- Bleomycin, V- Vincristine, DDoxorubicin

*** R- Rituximab, BM- Bendamustine.

\begin{tabular}{|c|c|c|c|}
\hline Characteristics & No. of Patients (\%) & Chi square Value with Degrees of Freedom & p \\
\hline Age & $10(33.3 \%)$ & & \\
<18 Years & $14(46.7 \%)$ & & \\
19-45 Years & $6(20 \%)$ & 2.981 & 0.084 \\
$>45$ Years & $20(66.7 \%)$ & & 0.06 \\
\hline Sex & $10(33.3 \%)$ & 7.5 & \\
Male & $14(46.7 \%)$ & & 0.038 \\
\hline Female & $16(53.3 \%)$ & & \\
Diagnosis & Table 1. Characteristics of Patients' Diagnosis & \\
\hline
\end{tabular}

\begin{tabular}{|c|c|}
\hline Characteristics & No. of Patients (\%) \\
\hline Treatment Protocol & $12(40 \%)$ \\
RCHOP & $14(46.7 \%)$ \\
ABVD & $4(13.3 \%)$ \\
R \& BM & $24(80 \%)$ \\
Relapse & $6(20 \%)$ \\
No & \\
\hline \multicolumn{2}{|c|}{ Table 2. Characteristics of Patients } \\
\hline
\end{tabular}

In our study, we found that association between FDG Uptake on PET Scan and relapse on histopathological examination was not significant at $p$ value of 0.084 . However, a large prospective study is recommended.

\section{DISCUSSION}

PET scan is generally performed at the time of initial diagnosis as a staging exam as well as a baseline examination prior to initiation of therapy. During therapy and particularly upon completion of therapy, PET/CT is useful in distinguishing viable tumour from necrosis or fibrosis. In general, PET/CT has a consistently high negative predictive value averaging about $85 \%$ across studies including patients with HL and/or diffuse large B-cell NHL.

The FDG, as an analogue of glucose, is taken up by cell membrane glucose receptors. Malignant tumours have a higher metabolic rate and generally express more numbers of specific membrane transporter proteins than normal cells and as an effect the FDG become trapped in these tumours cells. ${ }^{[3]}$ However, physiologic 18F-FDG uptake in Waldeyer's 
tonsillar ring is observed. Also, 18F-FDG may accumulate in inflammatory processes, followed by injury conditions, and benign tumours. Knowledge of these potentially false-positive lesions is essential for accurate FDG-PET/CT scan interpretation in oncologic patients.[11,12] Increased 18F-FDG uptake in inflammation may be explained by the recruitment of granulocytes, lymphocytes, and macrophages, which have enhanced levels of glucose transporters (GLUTs).[11] Infections in the head and neck region are common, as it may involve the sinonasal cavities, pharynx, tonsils, ears, mastoids, salivary glands, and dental tissues. Occasionally, it may be impossible to differentiate lymphoma from inflammation on the FDG-PET/CT scans. In these cases, it is necessary clinical, pathological, and imaging follow-up correlations.[5,11,12] Such as showed in our study, reactive tonsils and lymph nodes are responsible for decreased specificity of FDG-PET/CT scans for nodal or extranodal staging of many malignancies.[11]

Similar to our study, several studies have reported FDGPET/CT false-positives in patients with previous history of lymphoma. A 30-year-old, HIV-positive man, with a previous history of an atypical nasopharyngeal Burkitt lymphoma developed 18F-FDG avidity on the area of his initial disease. An open biopsy, however, revealed nodal RFH*.[6] Similar findings were observed by Beker et al.[2] in a 7-year-old child with Hodgkin lymphoma. The patient was considered cured, but 18F-FDG uptake in the abdomen was observed, which microscopically revealed RFH. To the best of our knowledge, there is only one case of palatine tonsil RFH showing $18 \mathrm{~F}$ FDG uptake in a 14-year-old boy after 6 cycles of aggressive chemotherapy for diffuse large B cell lymphoma.[8] It is interesting the fact that several RFH cases, usually affecting the tonsils, lymph nodes and thymus gland, in patients with haematological malignancies after chemotherapy have been reported. $[8,9,10,13]$ In these cases, it is often difficult to distinguish RFH from residual or recurrent disease, even if various imaging modalities are used. Furthermore, chemotherapy can lead to a diffusely increased 18F-FDG uptake within the whole bone marrow due to its activation. [5]

Biopsy is highly recommended to rule out a relapse or secondary transformation into aggressive lymphoma.

Comprehensive knowledge of physiologic and altered physiologic 18F-FDG uptake in the head and neck, as well as an awareness of potential pitfalls is necessary to minimize false-positive 18F-FDG results.

*RFH-Reactive Follicular Hyperplasia.

\section{CONCLUSIONS}

In our study of 30 patients, on post chemotherapy follow up, FDG uptake was present with SUV max of more than 5.0 in all patients, but only 6 patients had recurrence or relapse, proven histologically, post B/L tonsillectomy; rest of the patients had reactive hyperplasia. Thus, in this study, we found no significant relationship between increase FDG uptake in palatine tonsils in follow up cases of lymphoma, following histopathology results.

This study is hypothesis generating and a large prospective study to substantiate the findings is hence recommended.

\section{REFERENCES}

[1] Jerusalem G, Beguin Y, Fassotte MF, et al. Whole-body positron emission tomography using $18 \mathrm{~F}$ fluorodeoxyglucose for post-treatment evaluation in Hodgkin's disease and non-Hodgkin's lymphoma has higher diagnostic and prognostic value than classical computed tomography scan imaging. Blood 1999;94(2):429-33.

[2] Beker DB, Berrak SG, Canpolat C, et al. False positivity of FDG-PET/CT in a child with Hodgkin disease. Pediatr Blood Cancer 2008;50(4):881-3.

[3] Long NM, Smith CS. Causes and imaging features of false positives and false negatives on F-PET/CT in oncologic imaging. Insights Imaging 2011;2(6):679-98.

[4] Nakamoto Y, Tatsumi M, Hammoud D, et al. Normal FDG distribution patterns in the head and neck: PET/CT evaluation. Radiology 2005;234(3):879-85.

[5] Rosenbaum SJ, Lind T, Antoch G, et al. False-positive FDG PET uptake-the role of PET/CT. Eur Radiol 2006;16(5):1054-65.

[6] Bhargava P, Parker JA, Dezube BJ. Pitfalls of diagnosis based on abnormal flow cytometry and [(18) F] fluorodeoxyglucose positron emission tomography. Clin Lymphoma Myeloma 2008;8(2):117-20.

[7] Crocchiolo R, Fallanca F, Giovacchini G, et al. Role of 18FDG-PET/CT in detecting relapse during follow-up of patients with Hodgkin's lymphoma. Ann Hematol 2009;88(12):1229-36.

[8] Hosokai R, Imai C, Takachi T, et al. Reactive tonsillar enlargement with strong 18F-FDG uptake after chemotherapy for tonsillar diffuse large B-cell lymphoma. J Pediatr Hematol Oncol 2011;33(2):e87e8.

[9] Tokuc G, Oktem S, Genc A. Nasopharyngeal lymphoid hyperplasia after therapy for childhood lymphomas. Acta Oncol 2006;45(5):618-20.

[10] Kojima M, Murayama K, Higuchi K, et al. Reactive lymphoid hyperplasia with giant follicles associated with post-therapeutic state of hematological malignancies: a report of six cases. Leuk Lymphoma 2006;47(7):1404-6.

[11] Metser U Even-Sapir E. Increased (18) Ffluorodeoxyglucose uptake in benign, nonphysiologic lesions found on whole-body positron emission tomography/computed tomography (PET/CT): accumulated data from four years of experience with PET/CT. Semin Nucl Med 2007;37(3):206-22.

[12] Blodgett TM, Fukui MB, Snyderman $\mathrm{CH}$, et al. Combined PET-CT in the head and neck: part 1. Physiologic, altered physiologic and artifactual FDG uptake. Radiographics 2005;25(4):897-912.

[13] Oguz A, Karadeniz C, Citak EC, et al. Thymic and adenotonsillar enlargement after successful treatment of malignancies. Pediatr Hematol Oncol 2005;22(5):423-35. 